\title{
SALUD MENTAL Y CALIDAD DE VIDA AUTOPERCIBIDA EN ESTUDIANTES DE MEDICINA DE PARAGUAY.
}

\author{
SELF-PERCEIVED MENTAL HEALTH AND QUALITY OF LIFE IN MEDICAL \\ STUDENTS OF PARAGUAY.
}

Iván Barrios', Julio Torales².

\section{RESUMEN}

El objetivo de esta investigación fue estudiar la salud mental autopercibida como factor que puede influir en la calidad de vida autopercibida de estudiantes. Fue un estudio analítico de prevalencia, del que participaron 103 estudiantes de medicina de la Universidad Nacional de Asunción. Para la percepción de la salud mental se utilizó el cuestionario de Salud de Goldberg y para evaluar la calidad de vida autopercibida se utilizó la versión española del Short Form 36 Health Survey. Se encontró diferencia estadísticamente significativa entre el sexo y el dominio "dolor corporal". La edad se correlacionó significativamente con los dominios "Vitalidad" ( $r=-0,2 ; p=0,037)$, "Actividad emocional" ( $r=-0,2 ; p=0,34)$ y "Salud mental" $(r=-0,2 ; p=0,12)$. En el 28,2\% de los participantes se encontró presencia de psicopatología, siendo éstos quiénes presentaron una peor percepción de su calidad de vida. Estos datos impulsan a revisar las estrategias de prevención y promoción de la salud de los estudiantes.

\section{ABSTRACT}

The objective of this research was to study self-perceived mental health as a factor that could influence in students' self-perceived quality of life. It was an analytical study of prevalence, which involved 103 medical students of the National University of Asuncion. The Goldberg's Health Questionnaire was used for the perception of mental health and to assess the self-perceived quality of life it was used the Spanish version of the Short Form 36 Health Survey. It was found statistically significant difference between sex and "bodily pain" domain. Age was significantly correlated with "Vitality" ( $r=-0.2 ; p=0.037)$, "emotional activity" $(r=-0.2 ; p=0.34)$ and "mental health" $(r=$ $-0.2 ; p=0.12$ ) domains. In $28.2 \%$ of participants, the presence of psychopathology was found; being these who had a worse perception of their quality of life. These data drives to review strategies for prevention and health promotion of students.

\section{INTRODUCCIÓN}

a calidad de vida es un concepto amplio, in-

- fluido complejamente por la salud física del sujeto, por su estado psicológico y su nivel de independencia, por sus relaciones sociales, así como por la relación con los elementos esenciales de su entorno ${ }^{1,2,3}$. Se relaciona tanto con la salud física como con la salud mental del sujeto, y a través de la subjetividad del mismo, con aquello que es expresión de bienestar o malestar, más allá de lo que objetivamente ocurre en las interacciones de la persona con su ambiente 4.

Varios estudios se han realizado sobre la salud mental en estudiantes de medicina ${ }^{5,6}$, que han evidenciado altas prevalencias de trastornos mentales en esta población. Por lo tanto, se ha considerado importante, y se ha constituido en el principal objetivo de esta investigación, estudiar la salud mental autopercibida, así como características sociodemográficas particulares, como factores que puedan influir en la forma en que los estudiantes perciben su calidad de vida $^{3}$.

Los instrumentos de medición de la calidad de vida y la salud mental autopercibidas están formados por dominios o dimensiones que conforman un área de la conducta que se está estudiando y siguen, en su mayoría, el modelo
11 Estudiante universitario de Medicina y Cirugía. Facultad de Ciencias Médicas - Universidad Nacional de Asunción.

2 Profesor de Psiquiatría y Jefe del Departamento de Neurociencias, Facultad de Ciencias Médicas, Universidad Nacional de Asunción, San Lorenzo - Paraguay.

Correspondencia a: Nombre: Univ. Iván Barrios Facultad de Ciencias Médicas, Universidad Nacional de Asunción

San Lorenzo - Paraguay. ibarrios@neurociencias.org.py

Palabras clave: Salud mental, Calidad de vida, Estudiantes de medicina.

Keywords: Mental health, Quality of life, Medical students.
Procedencia y arbitraje: no comisionado, sometido a arbitraje externo.

Recibido para publicación: 05 de Abril del 2017 Aceptado para publicación: 25 de Junio del 2017

Citar como: Rev Cient Cienc Med 2017;20(1): 5 -10 
psicométrico ${ }^{7}$. En el presente estudio se utilizaron dos escales de fácil autoadministración, que se explican en detalle en la siguiente sección.

\section{MATERIALES Y MÉTODOS}

El presente fue un estudio analítico de prevalencia, con muestreo no probabilístico de casos consecutivos, que se realizó de mayo a junio del 2016. Participaron del mismo, estudiantes de medicina de la Universidad Nacional de Asunción de ambos sexos y que estén matriculados en el período académico 2016. Se incluyó a todos los estudiantes que aceptaron participar voluntariamente y se excluyó a aquellos con patologías crónicas de base. El tamaño de la muestra se calculó teniendo en cuenta una prevalencia de $58,2 \%$ de trastornos mentales ${ }^{5}$, un intervalo de confianza del $95 \%$, una desviación estándar de 1,96 y una amplitud de 0,10; el resultado fue de 93 sujetos. Finalmente, se incluyeron 103 sujetos.

Las variables independientes de estudio fueron fue la percepción de salud mental y calidad de vida; para evaluar la percepción de la salud mental, se utilizó el cuestionario de Salud de Goldberg (GHQ-12). Este es un instrumento elaborado por David Goldberg con el objetivo de evaluar niveles de salud mental autopercibidos en individuos o grupos de riesgo, y estimar la asociación entre la salud mental e indicadores de salud objetivos y con otras categorías psicosociales. Se presenta como una escala de tipo Likert (mejor que lo habitual, igual que lo habitual, menos que lo habitual, mucho menos que lo habitual). La puntuación se realiza concediendo 0 puntos a las primeras dos opciones y concediendo 1 punto a las últimas dos. La puntuación total es de 12 puntos. La categorización se realiza de la siguiente manera: entre 0 y 4 puntos obtenidos, se asume ausencia de psicopatología; entre 5 y 6 puntos, se considera una psicopatología subumbral; mientras que, entre 7 y 12 puntos, se establece que hay indicadores de presencia de psicopatología ${ }^{8}$.

La variable independiente fue la calidad de vida. Para evaluar la calidad de vida autopercivida se utilizó la versión española del Short Form 36 Health Survey (SF-36). El cuestionario de Salud SF-36 está compuesto por 36 preguntas que valoran los estados tanto positivos como negativos de la salud, así como 8 dimensiones que representan los conceptos de salud empleados con mayor frecuencia: funcionamiento físico, actividad física, dolor corporal, salud general, vitalidad, funcionamiento social, actividad emocional y salud mental del sujeto. Las puntuaciones de las 8 dimensiones del SF-36 están ordenadas de forma que a mayor valor mejor es el estado de salud. Para cada dimensión, los ítems son codificados, agregados y transformados en una escala con un rango de 0 (el peor estado de salud) a 100 (el mejor estado de salud). Lo anterior no se aplica a la dimensión dolor, donde el puntaje se interpreta de manera inversa: a mayor puntaje, menor dolor 9,10.

Los datos se analizaron utilizando el paquete de análisis estadísticos Epilnfo ${ }^{\circledR}$ versión 7.2 (CDC, Atlanta, Estados Unidos de América). Para la comparación entre variables cualitativas se usó Chi-cuadrado; para variables cuantitativas, se realizó la prueba de correlación de Pearson; para la comparación de medias, se utilizó t de Student y para comparar medianas, la prueba de Kruskal-Wallis. El nivel de significancia quedó establecido en $p<0,05$. En cuanto a los asuntos éticos, se obtuvo el consentimiento de los participantes usando el modelo de consentimiento informado de la Organización Mundial de la Salud. Todos los participantes fueron tratados igualmente, sin discriminación de sexo, raza, edad o cualquier otra característica. Los datos fueron confidenciales y solo se utilizaron para cumplir los objetivos de la investigación. El Departamento de Normas de la Dirección de Investigaciones de la Facultad de Ciencias Médicas de la Universidad Nacional de Asunción aprobó los aspectos éticos del presente estudio.

\section{RESULTADOS}

El coeficiente de alfa-Cronbach del SF-36 fue de 0,87 . En la tabla 1 se observan los estadísticos descriptivos de los dominios de calidad de vida. Se encontró diferencia estadísticamente significativa entre el sexo y el dominio "dolor corporal", ya que la media de las mujeres $(69,64)$ fue significativamente menor a la de los hombres $(82,72)(t=2,793[101] ; p=0,006)$. Por su parte, la edad se correlacionó significativamente con los dominios "Vitalidad" ( $r=-0,2 ; p=0,037)$, "Actividad emocional" $(r=-0,2 ; p=0,34)$ y "Salud mental" ( $r=-0,2 ; p=0,12)$.

Como puede observarse en la tabla 2, la "Vitalidad", "Actividad emocional" y "Salud mental" 
mostraron una asociación significativa con el curso en que se encontraban los estudiantes de medicina.

El coeficiente de alfa-Cronbach del GHQ-12 fue de 0,88 . En el $28,2 \%$ de los participantes se encontró presencia de psicopatología; en el $9,7 \%$, psicopatología subumbral; y, en el $62,1 \%$, ausencia de psicopatología. Entre los sujetos con psicopatología, el $42,4 \%$ se encontraba en quinto curso, y el $20,7 \%$, en cuarto curso y esto fue estadísticamente significativo (tabla 3).

En cuanto a la presencia de psicopatología como variable que pudiera estar relacionada con la calidad de vida, se observó que aquellos que presentaban psicopatología tenían una peor percepción de su calidad de vida con diferencias significativas en todos los dominios, excepto "dolor corporal" (Tabla 4).
Tabla 1. Descriptivos de los dominios de calidad de vida en los estudiantes

\begin{tabular}{lccc}
\hline \multicolumn{1}{c}{ Dominio } & Media & Desviación típica & Mediana \\
\hline Funcionamiento físico & 83,49 & 21,29 & 90 \\
Actividad física & 72,14 & 29,98 & 81,25 \\
Dolor corporal & 73,83 & 22,91 & 77,5 \\
Salud general & 60,58 & 21,76 & 65 \\
Vitalidad & 42,17 & 22,04 & 43,75 \\
Funcionamiento social & 65,77 & 29,12 & 75 \\
Actividad emocional & 59,06 & 31,89 & 66,66 \\
Salud mental & 55,83 & 23,78 & 60
\end{tabular}

Tabla 2. Dominios de calidad de vida y su relación con el curso

\begin{tabular}{|c|c|c|c|c|c|c|c|}
\hline \multirow{2}{*}{ Dominios } & \multicolumn{6}{|c|}{ Mediana } & \multirow{2}{*}{$\underset{\text { valor* }}{\mathbf{p}}$} \\
\hline & $10^{\circ+}$ & $2 .^{\circ}$ & $3 .^{\circ}$ & $4 .^{\circ}$ & $5 .^{\circ}$ & $6 .^{\circ}$ & \\
\hline $\begin{array}{l}\text { Funcionamiento } \\
\text { físico } \\
\text { Actividad física } \\
\text { Dolor corporal } \\
\text { Salud general } \\
\text { Vitalidad } \\
\text { Funcionamiento } \\
\text { social } \\
\text { Actividad } \\
\text { emocional } \\
\text { Salud mental }\end{array}$ & $\begin{array}{c}90 \\
75 \\
67,5 \\
60 \\
43,75 \\
75 \\
58,3 \\
60\end{array}$ & $\begin{array}{c}95 \\
81,25 \\
82,5 \\
67,5 \\
50 \\
75 \\
75 \\
72,5\end{array}$ & $\begin{array}{c}85 \\
87,5 \\
67,5 \\
65 \\
50 \\
62,5 \\
58,3 \\
50\end{array}$ & $\begin{array}{c}85 \\
75 \\
77,5 \\
65 \\
43,75 \\
87,5 \\
83,3 \\
65\end{array}$ & $\begin{array}{c}90 \\
75 \\
80 \\
65 \\
12,5 \\
37,5 \\
83,3 \\
25\end{array}$ & $\begin{array}{c}90 \\
93,75 \\
100 \\
90 \\
50 \\
87,5 \\
75 \\
60\end{array}$ & $\begin{array}{l}0,538 \\
0,759 \\
0,225 \\
0,747 \\
0,004 \\
0,174 \\
0,002 \\
0,009\end{array}$ \\
\hline
\end{tabular}

*Prueba Kruskal-Wallis + curso en que se encuentranan los estudiantes de medicina

\begin{tabular}{|c|c|c|c|c|}
\hline \multirow[b]{2}{*}{ Variables } & \multicolumn{3}{|c|}{ Salud mental autopercibida } & \multirow[b]{2}{*}{ p valor* } \\
\hline & $\begin{array}{c}\text { Psicopatología } \\
\text { n (\%) }\end{array}$ & $\begin{array}{c}\text { Subumbral } \\
\text { n (\%) }\end{array}$ & $\begin{array}{l}\text { Sano } \\
\text { n (\%) }\end{array}$ & \\
\hline Sexo & & & & 0,311 \\
\hline Masculino & $12(41,4)$ & $4(40)$ & $17(26,6)$ & \\
\hline Femenino & $17(58,6)$ & $6(60)$ & $47(73,4)$ & \\
\hline Curso & & & & 0,003 \\
\hline Primero & $4(13,8)$ & $4(40)$ & $19(29,7)$ & \\
\hline Segundo & $2(6,9)$ & $0(0)$ & $14(21,9)$ & \\
\hline Tercero & $5(17,2)$ & $2(20)$ & $12(18,8)$ & \\
\hline Cuarto & $6(20,7)$ & $1(10)$ & $12(18,8)$ & \\
\hline Quinto & $12(41,4)$ & $2(20)$ & $7(10,9)$ & \\
\hline Sexto & $0(0)$ & $1(10)$ & $0(0)$ & \\
\hline Diagnóstico previo & & & & 0,232 \\
\hline Sí & $9(31)$ & $2(20)$ & $10(15,6)$ & \\
\hline \multirow[t]{2}{*}{ No } & $20(69)$ & $8(80)$ & $54(84,4)$ & \\
\hline & & & \multicolumn{2}{|c|}{ * Prueba de Chi cuadra } \\
\hline
\end{tabular}


Tabla 4. Salud mental autopercibida y su asociación con la calidad de vida

\begin{tabular}{lcccc}
\hline \multirow{2}{*}{ Calidad de vida } & \multicolumn{3}{c}{ Salud mental autopercibida } & p valor* \\
\cline { 2 - 4 } & $\begin{array}{c}\text { Psicopatología } \\
\text { (DE) }\end{array}$ & $\begin{array}{c}\text { Subumbral } \\
\text { (DE) }\end{array}$ & $\begin{array}{c}\text { Sano } \\
\text { (DE) }\end{array}$ & p \\
\hline Funcionamiento físico & $68,96(29,71)$ & $87,50(21,50)$ & $89,45(11,82)$ & $\mathrm{p}<0,001$ \\
Actividad física & $48,49(36,23)$ & $86,87(22,71)$ & $80,56(20,83)$ & $\mathrm{p}<0,001$ \\
Dolor corporal & $68,36(29,08)$ & $77,50(30,27)$ & $75,74(17,98)$ & $\mathrm{p}>0,05$ \\
Salud general & $48,10(23,84)$ & $58(30,29)$ & $66,64(16,52)$ & $\mathrm{p}<0,001$ \\
Vitalidad & $20,90(19,64)$ & $37,50(20,62)$ & $52,53(15,29)$ & $\mathrm{p}<0,001$ \\
Funcionamiento social & $34,48(23,77)$ & $71,25(27,67)$ & $79,10(19,42)$ & $\mathrm{p}<0,001$ \\
Actividad emocional & $25(26,81)$ & $53,33(23,63)$ & $75,39(20,91)$ & $\mathrm{p}<0,001$ \\
Salud mental & $27,93(17,65)$ & $52(13,78)$ & $69,06(14,55)$ & $\mathrm{p}<0,001$ \\
\hline
\end{tabular}

*Prueba ANOVA

\section{DISCUSIÓN}

La idea que cada persona tiene sobre su calidad de vida está relacionada con las emociones, cogniciones y comportamientos que podrían tomarse como indicadores de riesgo de enfermedades y otros daños a la salud. La ansiedad, depresión, y otros trastornos psicopatológicos, pueden predisponer a la aparición de estrés, así como a cambios en el sistema inmune que repercute en la salud física y mental de las personas ${ }^{11}$.

El análisis de la calidad de vida que evalúa el SF-36 demuestra que la población estudiantil presenta el puntaje más alto en el dominio funcionamiento físico $(83,49 \pm 21,29)$ y el más bajo en el dominio vitalidad $(42,17 \pm 22,04)$. Para su interpretación se debe tener en cuenta que cero es el valor más bajo, que significa baja calidad de vida, y 100 el puntaje más alto, óptima calidad de vida. En cuanto al dolor corporal $(73,83 \pm 22,91)$ el puntaje se interpreta de manera inversa: a mayor puntaje menos dolor.

En cuanto a la variable sexo, se encontraron diferencias significativas entre hombres y mujeres en el dominio "dolor corporal", ya que las mujeres reportan menor calidad de vida relacionada con este dominio. Lo anterior podría explicarse ya que las mujeres están sometidas a mayores niveles de estrés ${ }^{12,13}$. El sexo no se relacionó con los otros dominios, lo que contrasta con otro estudio donde esta variable se relacionó con la salud general, la vitalidad y la salud mental ${ }^{5}$.

En nuestra muestra, la calidad de vida se relacionó con la edad, contrario a lo encontrado en estudio realizado con estudiantes de enfermería ${ }^{6}$. La vitalidad, por su parte, se relacionó con el curso de la carrera de Medicina y Cirugía al que estaban asistiendo los participantes; esto está en consonancia con estudios similares que informaron que la mejor percepción de la calidad de vida en este dominio se observó en estudiantes de primer curso ${ }^{7}$. En el presente estudio, el curso académico también se relacionó con la actividad emocional y la salud mental, observándose que la mayoría de los sujetos con psicopatología se encontraban en cuarto y quinto cursos de la carrera de Medicina. Esto podría deberse a que estos son los cursos de mayor carga académica de la carrera de Medicina en la Universidad Nacional de Asunción ${ }^{14}$.

En cuanto a salud mental autopercibida por los estudiantes de medicina, en este estudio se observó que la prevalencia de psicopatología encontrada fue de $28,2 \%$. Esto es bastante mayor al 18,5\% reportado en estudiantes de la Facultad de Medicina de la Universidad de Debrecen, Hungría 13, y también es mayor al 17\% de prevalencia de psicopatología encontrada en un estudio realizado en la Universidad de Edimburgo ${ }^{15}$.

La literatura evidencia que, al comparar grupos de estudiantes de medicina con otros grupos de edad similar, los primeros se exponen a mayores de niveles de ansiedad y depresión que otros estudiantes universitarios ${ }^{16}$. Se necesitan más investigaciones en este campo que incluyan variables como la personalidad y su relación con el desarrollo de patologías mentales; así como la identificación temprana de estos trastornos, a fin de garantizar que los estudiantes puedan acceder a mejores intervenciones y tratamientos oportunos, que aseguren buenos resultados ${ }^{14,17}$.

En esta investigación, la salud mental demostró que influye en la percepción de la calidad 
de vida en los estudiantes de medicina, ya que aquellos estudiantes con psicopatología tuvieron puntuaciones más bajas $y$, por lo tanto, una peor percepción de la calidad de vida. Lo anterior está en concordancia con estudios que demostraron que aquellas personas con resiliencia y buena salud mental tienen una mejor percepción de su calidad de vida ${ }^{18}$.

La medición de la calidad de vida de personas es actualmente un tema de interés debido a su importancia como factor pivotante de la salud mental de las personas y porque, desafortunadamente, en muchos círculos académicos todavía se la considera como algo no clínico. Sin embargo, extensivas revisiones de literatura han indicado que una pobre calidad de vida está asociada a distrés emocional y psicopatología ${ }^{19}$.

El presente estudio, aunque limitado, puede ayudar a sentar las bases de investigaciones mayores que corroboren estos resultados y que provean de evidencia útil para examinar la validez del contenido de diferentes herramientas de medición psicométrica. Asimismo, la presente investigación se enmarca y cobra gran relevancia en el contexto educacional actual de la Universidad Nacional de Asunción e impulsa a revisar las mallas curriculares de la carrera, los objetivos como institución, las políticas y estrategias de prevención y promoción de la salud integral de estudiantes. En este sentido, se considera como imperativo ya iniciar procesos de cambio con la aplicación de talleres e intervenciones psicológicas que pueden ayudar a que la Universidad Nacional de Asunción vaya logrando una nueva mirada de la educación, que no sólo enfatice la excelencia académica, sino también la salud mental, es decir, una formación más integral.

Finalmente, se contempla la necesidad de crear programas e instancias de protección, para que los estudiantes universitarios puedan distraerse de los estudios de manera sana, sintiéndose parte de una red social que se preocupa por su bienestar psicológico. Por ejemplo, se podría disponer de talleres artísticos (músi$\mathrm{ca}$, pintura, danza, manualidades, entre otros) y actividades deportivas, en los cuales los estudiantes de la carrera de Medicina y Cirugía aprovechen sus destrezas manuales (y físicas) para distraerse del trabajo académico y, a la vez, utilicen su hemisferio derecho. Esta zona del cerebro, donde se ubican la intuición y la creatividad recibe las informaciones globales, de forma no verbal, y recoge una realidad más vasta y próxima al mundo sensorial y afectivo ${ }^{20}$.

\section{REFERENCIAS}

1. Henning MA, Krägeloh CU, Hawken SJ, Zhao Y, Doherty I. THE QUALITY OF LIFE OF MEDICAL STUDENTS STUDYING IN NEW ZEALAND: A COMPARISON WITH NONMEDICAL STUDENTS AND A GENERAL POPULATION REFERENCE GROUP. Teach Learn Med. 2012. 24 (4); 334-40. Disponible en: https://www. ncbi.nlm.nih.gov/pubmed/23036001

2. Henning MA, Hawken SJ, Hill AG. THE QUALITY OF LIFE OF NEW ZEALAND DOCTORS AND MEDICAL STUDENTS: WHAT CAN BE DONE TO AVOID BURNOUT?. N Z Med J. 2009. 122 (1307); 102-10. Disponible en: https:// www.ncbi.nlm.nih.gov/pubmed/20148052

3. Grimaldo M. CALIDAD DE VIDA EN ESTUDIANTES DE SECUNDARIA DE LA CIUDAD DE LIMA. Av Psicol. 2012. 20 (1); 89-102. Disponible en: https://www.researchgate.net/ publication/234015995_CALIDAD_DE_VIDA_ EN_ESTUDIANTES_DE_SECUNDARIA_DE_LA_CIUDAD_DE_LIMA_Life_quality_in_high_school_students_of_Lima_city

4. Gutiérrez $M$, Montoya $L$, Toro B, Briñón $M$, Rosas E, Salazar L. DEPRESIÓN EN ESTUDIANTES UNIVERSITARIOS Y SU ASOCIACIÓN CON EL ESTRÉS ACADÉMICO. CES
Med. 2010. 24 (1); 7-17. Disponible en: http:// www.scielo.org.co/scielo.php?script=sci_arttext\& pid $=$ S0120-87052010000100002

5. Blázquez JJ, González JC, Cano-Montero EI, Gutiérrez S. CALIDAD DE VIDA UNIVERSITARIA: IDENTIFICACIÓN DE LOS PRINCIPALES INDICADORES DE SATISFACCIÓN ESTUDIANTIL. Rev Educ. 2013. (362), 458-84. Disponible en: https://dialnet.unirioja.es/servlet/ar ticulo;jsessionid=216E617B1CF7FE52A713901A 270249F1. dialnet01? codigo $=4480193$

6. Hidalgo-Rasmussen CA, Hidalgo-San Martín A, Rasmussen-Cruz B, Montaño-Espinoza R. CALIDAD DE VIDA, SEGÚN PERCEPCIÓN Y COMPORTAMIENTOS DE CONTROL DEL PESO POR GÉNERO, EN ESTUDIANTES UNIVERSITARIOS ADOLESCENTES EN MÉXICO. Cad Saúde Pública. 2011. 27 (1); 67-77. Disponible en: http://www.scielo.br/scielo.php?pid=S0102$311 \times 2011000100007 \&$ script $=s c i$ abstract\&tlng=es

7. Arostegui I, Núñez-Antón VA. ASPECTOS ESTADÍSTICOS DEL CUESTIONARIO DE CALIDAD DE VIDA RELACIONADA CON SALUD SHORT FORM-36 (SF-36). Estad Esp. 2008. 50 (167); 147-92. Disponible en: https://dialnet.uni- 
rioja.es/servlet/articulo?codigo $=2593867$

8. Garmendia ML. ANÁLISIS FACTORIAL: UNA APLICACIÓN EN EL CUESTIONARIO DE SALUD GENERAL DE GOLDBERG, VERSIÓN DE 12 PREgUNTAS. Rev Chil Salud Pública. 2007. 11 (2); 57-65. Disponible en: http://www. revistasaludpublica.uchile.cl/index.php/RCSP/article/view/3095

9. Pacheco M, Michelena M, Mora R, Miranda O. CALIDAD DE VIDA RELACIONADA CON LA SALUD EN ESTUDIANTES UNIVERSITARIOS. Rev Cuba Med Mil. 2014. 43 (2); 157-68. Disponible en: $h t t p: / / s c i e l o . s / d . c u / s c i e l o . p h p ? s c r i p t=s c i$ arttext\&pid=S0138-65572014000200004

10. Vilagut G, Ferrer M, Rajmil L, Rebollo P, Permanyer-Miralda G, Quintana JM, et al. EL CUESTIONARIO DE SALUD SF-36 ESPAÑOL: UNA DÉCADA DE EXPERIENCIA Y NUEVOS DESARROLLOS. GaC Sanit. 2005. 19 (2); 135-50. Disponible en: http:// www.scielo.cl/scielo.php?script=sci_arttext\&pid =S0717-95532003000200002

11. Abdulghani HM, AlKanhal AA, Mahmoud ES, Ponnamperuma GG, Alfaris EA. STRESS AND ITS EFFECTS ON MEDICAL STUDENTS: A CROSS-SECTIONAL STUDY AT A COLLEGE OF MEDICINE IN SAUDI ARABIA. J Health Popul Nutr. 2011. 29 (5); 516-22. Disponible en: $\quad$ https://www.ncbi.nlm.nih.gov/pmc/articles/ PMC3225114/

12. Tempski P, Perotta B, Pose R, Viera J. A QUESTIONNAIRE ON THE QUALITY OF LIFE OF MEDICAL STUDENTS. Med Educ. 2009. 43 (11); 1107-8. Disponible en: http://onlinelibrary. wiley.com/doi/10.1111/j.1365-2923.2009.03476.x/ epdf

13. Bíró É, Balajti I, Ádány R, Kósa K. DETERMINANTS OF MENTAL WELL-BEING IN MEDICAL STUDENTS. Soc Psychiatry Psychiatr Epidemiol. 2009. 45 (2); 253-8.

14. Torales-Benítez J, Girala N, Moreno-Giménez M, Arce A, Trinidad S, Estigarribia E, et al. DEPRESIÓN Y ANSIEDAD EN ESTUDIANTES
DE MEDICINA DE LA UNIVERSIDAD NACIONAL DE ASUNCIÓN. Rev Paraguaya Psiquiatr. 2013. 1 (1); 12-28.

15. Carson AJ, Dias S, Johnston A, McLoughlin MA, O'Connor M, Robinson BL, et al. MENTAL HEALTH IN MEDICAL STUDENTS. A CASE CONTROL STUDY USING THE 60 ITEM GENERAL HEALTH QUESTIONNAIRE. Scott Med J. 2000. 45 (4); 115-6.

16. Von Bothmer MIK, Fridlund B. SELF-RATED HEALTH AMONG UNIVERSITY STUDENTS IN RELATION TO SENSE OF COHERENCE AND OTHER PERSONALITY TRAITS. Scand $J$ Caring Sci. 2003. 17 (4); 347-57.

17. Barrios I, Miltos V, Piris A, Piris G, Ramírez C, Rodríguez J, et al. TAMIZAJE DE SALUD MENTAL MEDIANTE EL TEST M.I.N.I. EN ESTUDIANTES DEL CICLO BÁSICO DE MEDICINA DE LA UNIVERSIDAD NACIONAL DE ASUNCIÓN. An Fac Cienc Médicas. 2015. 48 (1); 59-68.

18. Salinero-Fort MÁ, Gómez-Campelo $P$, Bragado-Alvárez C, Abánades-Herranz JC, Jiménez-García $R$, Burgos-Lunar $C$ de, et al. HEALTH-RELATED QUALITY OF LIFE OF LATIN-AMERICAN IMMIGRANTS AND SPANISH-BORN ATTENDED IN SPANISH PRIMARY HEALTH CARE: SOCIO-DEMOGRAPHIC AND PSYCHOSOCIAL FACTORS. PLOS ONE. 2015. 10 (4); e0122318.

19. Connell J, Brazier J, O'Cathain A, LloydJones M, Paisley S. QUALITY OF LIFE OF PEOPLE WITH MENTAL HEALTH PROBLEMS: A SYNTHESIS OF QUALITATIVE RESEARCH. Health Qual Life Outcomes. 2012. 10; 138.

20. Barros P, Matamorros M. PERCEPCIÓN DE CAMBIO ATRIBUIDA AL PROCESO DE ASISTENCIA PSICOLÓGICA HUMANISTA BREE EN ESTUDIANTES DE LA FACULTAD DE MEDICINA DE LA UNIVERSIDAD DE CHILE. Tesis de Grado. Universidad de Chile. 2009. Disponible en: http://www.tesis.uchile.cl/tesis/uchile/2009/ cs-barros_p/html/index-frames.html 